\title{
The Preparation of Matrine Liposome and Its Antiglioma Activity Study
}

\author{
Shao-Rong Han, Hai Gong, Yan-Ming Wang, Xiao-Yan Lv, Cong Zhang, An-Na Tong, \\ Guang-Hui Yuan, and Bao-Yi Zhang
}

Department of Radiation Oncology, Jinan Military General Hospital, Jinan 250031, China

Correspondence should be addressed to Yan-Ming Wang; wangym0369@126.com

Received 3 May 2014; Accepted 18 June 2014; Published 14 July 2014

Academic Editor: Tanaji Talele

Copyright (c) 2014 Shao-Rong Han et al. This is an open access article distributed under the Creative Commons Attribution License, which permits unrestricted use, distribution, and reproduction in any medium, provided the original work is properly cited.

\begin{abstract}
The aim of the study was to study the preparation of matrine liposome and its activity for resisting cells, to study the preparation of matrine liposome by orthogonal design, and to observe the inhibiting effect of matrine on glioma through MTT method, Flow Cytometer, and electron microscope. The results showed that we take the encapsulation efficiency as the index; the optimal preparation of matrine liposome is $100 \mathrm{mg}$ of lecithin, $40 \mathrm{mg}$ of cholesterol, phosphate buffered saline (PBS) with pH value 6.4 , and $40 \mathrm{mg}$ of matrine. The result of MTT testing is shown that the tested group with medium and high dosage $(0.75,1.0,1.25 \mathrm{mg} / \mathrm{L})$ of matrine has significant inhibiting rate to the growth of BT 325 cells. The $A$ value in tested group with medium and high dosage is up to $0.19 \pm 0.03$. The methods of Flow Cytometer and electron microscope prove that matrine can inhibit the growth of BT 325 cells. Matrine is active in inhibiting brain glioma.
\end{abstract}

\section{Introduction}

Sophora flavescens is the dried root of Sophora flavescens Ait; it has many other names such as Kugu, Chuansheng, Phoenix Talon, Niushen, and Dikui and grows all over our nation. It is first recorded in Shen Nong's Herbal Classic and listed as a medicine, which is the common traditional Chinese medicine with the efficacy of cleaning heat, insecticide, diuresis, and so forth. It applies to heat dysentery, hemafecia, jaundice, anuresis, leucorrhea with reddish discharge, pain and tickle in pudendum, eczema, tickle in skin tumor, mange, and leprosy, and it is also used for external treating of trichomonas vaginitis. Modern research suggests that Sophora flavescens contain multiple chemical constituents and possess lots of biologically active ingredients. The main chemical constituents are alkaloid and flavonoids compounds; besides, it contains dialkyl group chromone, quiniones, triterpenoid saponins, and so forth, [1-4]. Modern pharmacological research proves that Sophora flavescens has the effect of antiviral hepatitis and antiliver damage, antitumor, antifibrosis, antiarrhythmia, antimicrobial, regulation of the immune, and so forth, $[5,6]$.
In order to increase the availability of matrine in liver and prepare the matrine liposome in accordance with the requirements of liver targeting, this experiment studies the preparation technology of matrine liposome; meanwhile, we discuss its inhibiting function to glioma.

\section{Experimental}

Rotary evaporator comes from Shanghai Yarong Biochemical Instrument Factory; high efficiency capillary electrophoresis (HPCE) is from BECKMAN instrument company, American BDFACSAria of Flow Cytometer; super-clean bench is from Suzhou Purification Equipment Factory; $\mathrm{CO}_{2}$ incubator is from American NBS company; MTT is purchased from Beijing Solarbio Science \& Technology Co., Ltd.; RPMI 1640 cells culture medium is purchased from American Gibco company; propidium iodide (PI) is from Amersco company.

2.1. Reagent and Cell Strain. The purity of Matrine is $98.4 \%$ Which is prepared in Laboratory. Reference substance of matrine is purchased from Institute for the Control of 
Chinese Pharmaceutical and Biological Product. BT 325 cells are sent by Chinese Medical Sciences University.

2.2. The Preparation of Liposome. It is prepared by reservephase evaporation method. Firstly, lecithin and cholesterol are weighed according to a certain ratio, and then they are put into pear-shaped flask together with 10 mg of PEG 2000DSPE and $0.1 \mathrm{mg}$ of vitamin E and are soluble in chloroform. And then, we take the appropriate matrine and dilute it to $10 \mathrm{~mL}$ with pH 7.4 of PBS. After that, put this biphasic system into the water-bath type ultrasonic apparatus for 5 minutes of high power ultrasound. And then, place them in indoor temperature for 30 minutes; there should be no delamination in water. After doing this, put the mixture into rotary evaporator with $28^{\circ} \mathrm{C}$ of water bath; next, steam the organic solvent by pressure reduction, and then, it can be processed by ultrasonic for a short time. The finished products are put into $4^{\circ} \mathrm{C}$ of refrigerator for cold storage.

\subsection{Determination of Encapsulation Efficiency}

Separating Conditions. Running voltage is $30 \mathrm{kV}$; detection wavelength is $206 \mathrm{~nm}$; sample injection method is pressure sampling; quantity of sample injection is $0.5 \mathrm{Psi} \times 5 \mathrm{~s}$; temperature is $25^{\circ} \mathrm{C}$ :

$$
\text { encapsulation efficiency }=\frac{W_{\text {total }}-W_{\text {free }}}{W_{\text {total }}} \times 100 \% \text {, }
$$

where $W_{\text {total }}$ represents total medicine and $W_{\text {free }}$ represents free medicine.

The Preparation of Standard Curve. First, we take matrine reference substance with different concentration $(0.5,1.0$, $1.5,2.0,4.0$, and $8.0 \mathrm{mg} / \mathrm{mL}$ ) and then make the HPEC measurement with the separating condition above. We use concentration peak area of matrine to do a linear regression analysis; the results show that the linear relation is good under tested concentration. The standard curve values for matrine reference substance were " $y=29538 x+7132, R^{2}=0.9999$ ".

2.4. Orthogonal Experimental Method. According to preexperimental condition, we take the dosage of lecithin $(A)$, dosage of cholesterol $(B), \mathrm{pH}$ value of phosphate buffered saline (PBS) $(C)$, and dosage of matrine $(D)$ as the factors to consider, and we take the encapsulation efficiency as the index. Then, take 3 levels for each factor; we make this orthogonal experimental design by adopting $\mathrm{L}_{9}\left(3^{4}\right)$ orthogonal table (see Tables 1 and 2).

2.5. BT 325 Cells Culture. According to the traditional way, we culture the BT 325 cells in culture media which have $10 \%$ of calf serum ( $9 \mathrm{~mL}$ of DMEM, $10 \mathrm{~mL}$ of calf serum, $0.1 \mathrm{~g}$ of penicillin, and $0.1 \mathrm{~g}$ of streptomycin are contained in each $100 \mathrm{~mL}$ ). The temperature of incubator is $37^{\circ} \mathrm{C}$ with $98 \%$ of relative humidity and $5 \%$ of $\mathrm{CO}_{2}$. Single-layer of cells grows against the wall; when they grow till $80 \%$ to $90 \%$, cells are merging, trypsin enzyme-digesting cells are subcultured, and logarithmic growth phase cells are collected for experiment.
TABLE 1: Orthogonal factor level table.

\begin{tabular}{lcccc}
\hline Level & $A(\mathrm{mg})$ & $B(\mathrm{mg})$ & $C(\mathrm{pH})$ & $D(\mathrm{mg})$ \\
\hline 1 & 100 & 25 & 6.4 & 20 \\
2 & 80 & 40 & 6.8 & 30 \\
3 & 60 & 60 & 7.0 & 40 \\
\hline
\end{tabular}

TABLE 2: Orthogonal experiment result of matrine liposome.

\begin{tabular}{lccccc}
\hline Test number & $A$ & $B$ & $C$ & $D$ & $\begin{array}{c}\text { Significant } \\
\text { influence (\%) }\end{array}$ \\
\hline 1 & 1 & 1 & 1 & 1 & 18.32 \\
2 & 1 & 2 & 2 & 2 & 34.64 \\
3 & 1 & 3 & 3 & 3 & 46.38 \\
4 & 2 & 1 & 2 & 3 & 14.37 \\
5 & 2 & 2 & 3 & 1 & 22.12 \\
6 & 2 & 3 & 1 & 2 & 15.46 \\
7 & 3 & 1 & 3 & 2 & 16.94 \\
8 & 3 & 2 & 1 & 3 & 52.14 \\
9 & 3 & 3 & 2 & 1 & 28.46 \\
\hline$k 1$ & 33.113 & 16.543 & 28.64 & 22.967 & \\
$k 2$ & 17.317 & 36.3 & 25.823 & 22.347 & \\
$k 3$ & 32.513 & 30.1 & 28.48 & 37.63 & \\
$R$ & 15.796 & 19.757 & 2.817 & 15.283 & \\
\hline
\end{tabular}

2.6. Determination of the Influence of Matrine on BT 325 Cell Proliferation by MTT Method. BT 325 cells in log phase are abstract to digest by pancreatin; after that, the cell concentration is adjusted to $1 \times 10^{5} / \mathrm{mL}$, and then they are cultured in 96 pore plates with $100 \mu \mathrm{L}$ for each pore. $0.25,0.5,0.75,1.0$, and $1.25 \mathrm{mg} / \mathrm{L}$ of matrines are added and 6 parallel pores are set for each concentration. The culture media of same volume are added in control group, and then the culture is continued in incubator with $37^{\circ} \mathrm{C}$ and $5 \% \mathrm{CO}_{2}$ for 72 hours; after that, the supernatant is discarded. Twenty $\mu \mathrm{L}$ MTT solution with concentration of $0.5 \mathrm{mg} / \mathrm{mL}$ is added in each pore, and then the culture is continued in incubator with $37^{\circ} \mathrm{C}$ and $5 \% \mathrm{CO}_{2}$ for 4 hours; after that, the supernatant is discarded, $100 \mu \mathrm{L}$ DMS are added in each pore, and then oscillation and mixing are taken. After this, we use enzyme reader to determine the absorbance $(A)$ under the condition of $570 \mathrm{~nm}$ wavelength and then calculate the cells inhibiting rate. Consider

Inhibiting rate $=(A$ value of control group

$$
\begin{aligned}
& -A \text { value of medicated group }) \\
& \times(A \text { value of control group })^{-1} \text {. }
\end{aligned}
$$

2.7. Flow Cytometer. BT 325 cells which have been cultured for 72 hours in matrine treatment group and in control group are collected, respectively, and the cells number is adjusted to $1 \times 10^{8} / \mathrm{mL}$; they are washed twice by PBS, and $75 \%$ of ethanol is added (absolute ethyl alcohol is prepared by PBS), and then the single-cell suspension is made. We store this suspension overnight under $4^{\circ} \mathrm{C}$ temperature. And we take 
TABLE 3: Table of variance analysis.

\begin{tabular}{|c|c|c|c|c|}
\hline Factor & Sum of squares of deviations & Freedom degree & $F$ ratio & $F$ critical value \\
\hline Dosage of lecithin & 480.833 & 2 & 1.071 & 19.000 \\
\hline Dosage of cholesterol & 612.549 & 2 & 1.364 & 19.000 \\
\hline Phosphate buffered saline with $\mathrm{pH}$ values & 15.017 & 2 & 0.033 & 19.000 \\
\hline Dosage of matrine & 448.978 & 2 & 1.000 & 19.000 \\
\hline Error & 448.98 & 2 & & \\
\hline
\end{tabular}

the centrifugation to remove ethanol, and we wash them twice by PBS and add $20 \mu \mathrm{L}$ RNas which contain $5 \mu \mathrm{g} / \mathrm{mL}$ PI and $1 \mathrm{mg} / \mathrm{mL}$; then, we make the PI staining and hatch in the dark place for 30 minutes under $37^{\circ} \mathrm{C}$ temperature. After that, we filter them 5 times by Nylon net with 300 meshes, and finally we determine the cell cycle distribution.

2.8. Observation of Transmission Electron Microscope. We abstract the above BT 325 cells which have been treated by matrine and fix them by $1 \%$ osmic acid after washing and then wash them with $0.1 \%$ PBS. Then, the cell morphology is observed by transmission electron microscope, after normal dehydration, penetration, epoxy resin embedding. and section treatments.

2.9. Statistical Treatment. Each measuring data are expressed by $\bar{x} \pm s$. Data among groups are analyzed by single factor variance and contrasted by $t$-test.

\section{Results and Discussion}

3.1. Result of Orthogonal Experiment. This experiment adopts orthogonal experiment method, takes encapsulation efficiency as the index, and takes the dosage of lecithin, dosage of cholesterol, phosphate buffered saline with $\mathrm{pH}$ values, and dosage of matrine as the factors to consider; then, take 3 levels for each factor and do this experiment according to the orthogonal experimental table. From the range analysis of this result, we can see that factor $B$ (dosage of cholesterol) has the greatest influence on this result, followed by factor $A$ (dosage of lecithin). The optimal abstract technique is A1B2C1D3, namely, $100 \mathrm{mg}$ of lecithin, $40 \mathrm{mg}$ of cholesterol, $\mathrm{pH} 6.4 \mathrm{of}$ phosphate buffered saline (PBS), and $40 \mathrm{mg}$ of matrine.

The result of variance analysis shows that factor $B$ has the most significant influence on encapsulation efficiency, factors $A$ and $D$ have obvious influence, and factor $C$ has the lowest impact. Thus, the conclusion of variance analysis is consistent with direct analysis (see Table 3).

3.2. Determine Result of MTT Method. Compared with control group, $A$ value of the tested group with low dosage ( 0.25 , $0.5 \mathrm{mg} / \mathrm{L}$ ) has no obvious changes and thus has no statistical significance; the matrine tested group with medium and high dosage has significantly differences in the growth inhibition rate of BT 325 cells, among which the $A$ value of matrine tested group with high dosage $(1.25 \mathrm{mg} / \mathrm{L})$ goes up to $0.19 \pm$ 0.03 , which indicate that matrine can inhibit the growth of glioma BT 325 cells in different degree and present on certain dose dependant manner (see Table 4).
TABLE 4: The growth inhibiting rate of matrine to BT 325 cells.

\begin{tabular}{lc}
\hline Different group & $A$ Value \\
\hline Control group & $0.32 \pm 0.04$ \\
$0.25 \mathrm{mg} / \mathrm{L}$ & $0.31 \pm 0.03$ \\
$0.5 \mathrm{mg} / \mathrm{L}$ & $0.30 \pm 0.04$ \\
$0.75 \mathrm{mg} / \mathrm{L}$ & $0.28 \pm 0.04$ \\
$1.0 \mathrm{mg} / \mathrm{L}$ & $0.24 \pm 0.04$ \\
$1.25 \mathrm{mg} / \mathrm{L}$ & $0.19 \pm 0.03$ \\
\hline
\end{tabular}

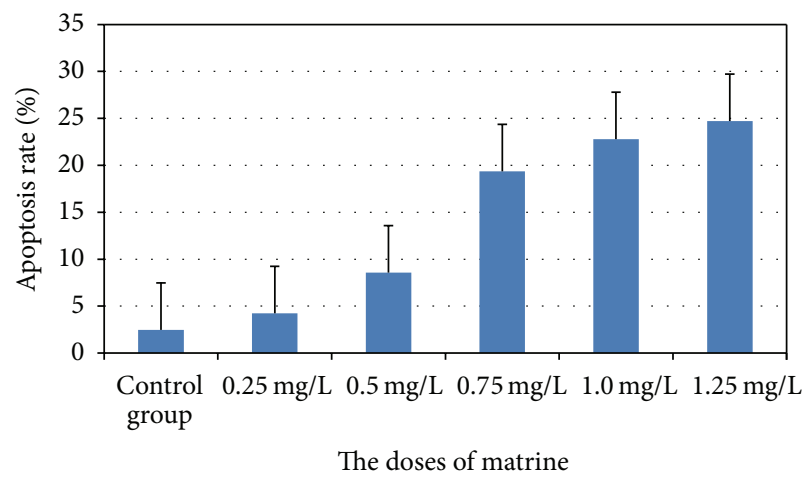

FIGURE 1: The influence of matrine with different concentration on apoptosis rate of BT 325 cells.

3.3. Result Analysis of Flow Cytometer. From Figure 1, we know that, after treating BT 325 cells by various concentrations of matrine for 72 hours, BT 325 cells apoptosis in different degree, among which the apoptosis cells in low dose $(0.25 \mathrm{mg} / \mathrm{L})$ group account for $2.47 \%$ of total cells and thus have no statistical significance. Apoptosis rate in remaining groups is high, and the apoptosis cells in high dose $(1.25 \mathrm{mg} / \mathrm{L})$ group account for $24.71 \%$ of total cells.

3.4. Result of Transmission Electron Microscope. Observing under the electron microscope, we can see that the shape of BT 325 cells in control group is regular and generally presents on elliptical or orbicular-ovate with clear nuclear membrane, and much euchromatin and less heterochromatin exist in nuclear and have clear ridge structure, a few of rough endoplasmic reticulum, completed structure, and uniform matrix. After being treated by matrine $(1.25 \mathrm{mg} / \mathrm{L})$ for 72 hours, the cells shrink, the volume reduces, the nucleolus reduces and concentrates, the nuclear chromatin concentrates and marginalizes, and some organelle, ribosome, and nuclear fragments are parceled by cell membrane and form 


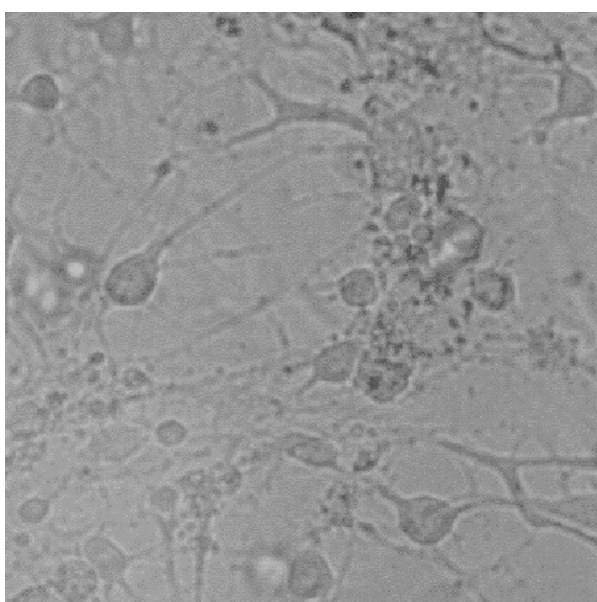

(a) State of normal cell

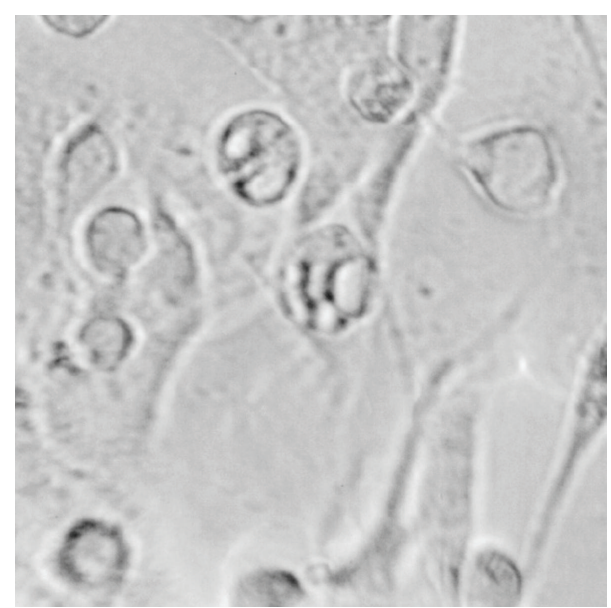

(b) State of cells treated with the matrine $(1.25 \mathrm{mg} / \mathrm{L})$

FIGURE 2: Cell morphology under the electron microscope.

apoptosis body; then, sprout falls from cells surface. There are a lot of vacuoles with various sizes in cytoplasm, all of which are fully compliant with the morphology manifestation of cell apoptosis. This is shown in Figure 2.

\section{Discussion}

Tumor etiology studies show that the occurrence and development of tumor are closely related to rapid growth of tumor cells and antiapoptotic mechanisms [7]. Nervous system tumor, including the growth and inhibition of Nervous glioma, has close relationship with cell apoptotic [8].

The same as cell proliferation, the cell apoptotic is also a concrete process which is controlled by gene. There are main two paths; one is to activate Caspase through the death receptors on cell membrane and the other is to release the cell apoptosis factor through the pathway of mitochondria in cytoplasm to activate Caspase. These active Caspases could be able to effect the important protein to make the normal cells apoptotic. If treated by medicine, the malignant tumor could be shrink and even apoptotic; this is mainly because the medicine can promote the apoptotic of tumor cells and inhibit its proliferation [9].

Brain glioma is the most common primary tumor in intracranial. Because of its high sickness rate and difficulty in operating due to its location, it presents on high mortality rate in treatment, and the treatment effect after operation is poor. We can classify the brain glioma into four types which are low degree of malignant tumor, frontal glioma, glioma with astrocytoma phenotype, and glioblastoma, according to its damaged condition to organism and difficulty in treatment. The traditional treatment mainly adopts triple therapy which connects the microneurosurgical operation with chemotherapy and radiotherapy, because the glioma is insensitive to chemotherapy and radiotherapy and develops the drug resistance. The survival rate of patients who have this disease for 5 years is not high [10].

This study determines the inhibiting effect of matrine with different concentration on glioma by MTT method. The results prove that the matrines with media and high dose have the significant differences in growth inhibiting rate of BT 325 cells, which indicate that matrine can inhibit the growth of glioma BT 325 cells in different degree and present on certain dose dependant manner. Flow Cytometer suggests that, after treating BT 325 cells by various concentrations of matrine for 72 hours, it makes BT 325 cells apoptotic to some degree. When observing under the electron microscope, we can see that the shape of BT 325 cells in control group is regular and generally presents on elliptical or orbicularovate with clear nuclear membrane, and much euchromatin and less heterochromatin exist in nuclear and have clear ridge structure, a few of rough endoplasmic reticulum, completed structure, and uniform matrix. After being treated by matrine $(1.25 \mathrm{mg} / \mathrm{L})$ for 72 hours, the cells shrink, the volume reduces, the nucleolus reduces and concentrates, the nuclear chromatin concentrates and marginalizes, and some organelle, ribosome, and nuclear fragments are parceled by cell membrane and form apoptosis body, and sprout fall from cells surface; there are a lot of vacuoles with various sizes in cytoplasm, all of which are fully compliant with the morphology manifestation of cell apoptosis.

\section{Conflict of Interests}

The authors declare that there is no conflict of interests regarding the publication of this paper.

\section{Authors' Contribution}

Shao-Rong Han and Hai Gong are equally contributed to this paper.

\section{References}

[1] J. Yuan, Z. J. Zhang, and B. Cong, "Bioactivity of matrine and its research development," Pesticide, vol. 42, no. 7, pp. 1-4, 2003.

[2] L. J. Wu, T. Miyase, A. Ueno, M. Kuroyanagi, T. Noro, and S. Fukushima, "Studies on the constituents of Sophora flavescens 
AITON. II," Chemical \& Pharmaceutical Bulletin, vol. 33, no. 8, pp. 3231-3236, 1985.

[3] Y. Y. Zhao and R. Y. Zhang, "Review of study on chemical composition of matrine," Researches and Development of Natural Products, vol. 3, no. 3, pp. 93-102, 1991.

[4] A. Yagi, M. Fukunaga, and N. OkuZako, "Antifungal substances from Sophora flavesecns," Shoyakugaku Zasshi, vol. 43, no. 4, pp. 343-346, 1989.

[5] W. Z. Kang, Y. M. Xie, and Q. H. Nie, "Study on preventive effect of matrine on experimental hepatic fibrosis," World Journal of Digestion, vol. 11, no. 2, pp. 195-198, 2003.

[6] H. Y. She, X. H. Wu, S. J. Gong, J. M. Zhang, and Z. Z. Feng, "Matrine injection promote archusia and effect of curing chronic grave hepatitis," Modern Practical Medicine, vol. 13, no. 9, pp. 436-438, 2001.

[7] T. J. McDonnell, R. E. Meyn, and L. E. Robertson, "Implications of apoptotic cell death regulation in cancer therapy," Seminars in Cancer Biology, vol. 6, no. 1, pp. 53-60, 1995.

[8] O. Micheau, E. Solary, A. Hammann, F. Martin, and M. Dimanche-Boitrel, "Sensitization of cancer cells treated with cytotoxic drugs to Fas-mediated cytotoxicity," Journal of the National Cancer Institute, vol. 89, no. 11, pp. 783-789, 1997.

[9] D. Schiffer, P. Cavalla, A. Migheli et al., "Apoptosis and cell proliferation in human neuroepithelial tumors," Neuroscience Letters, vol. 195, no. 2, pp. 81-84, 1995.

[10] F. B. Furnari, T. Fenton, R. M. Bachoo et al., "Malignant astrocytic glioma: genetics, biology, and paths to treatment," Genes and Development, vol. 21, no. 21, pp. 2683-2710, 2007. 

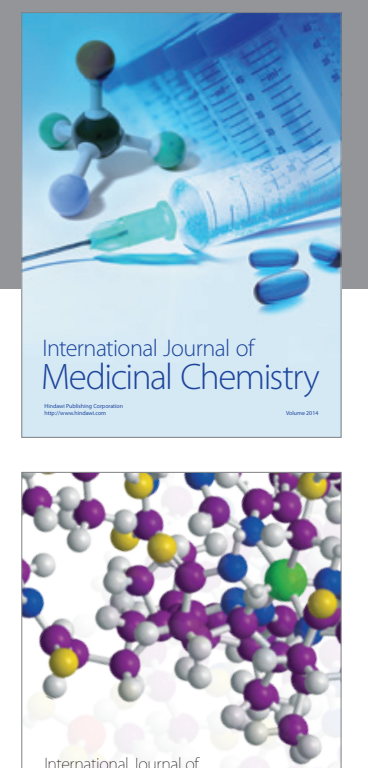

\section{Carbohydrate} Chemistry

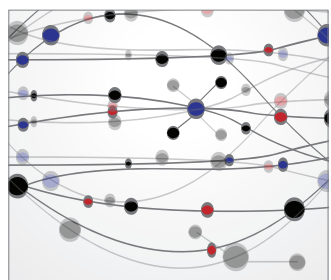

The Scientific World Journal
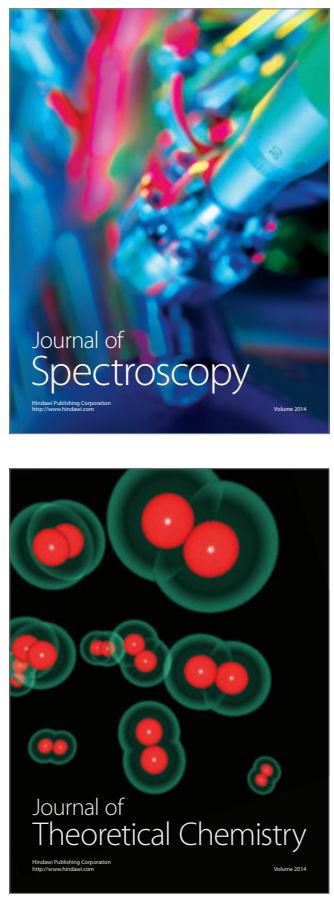
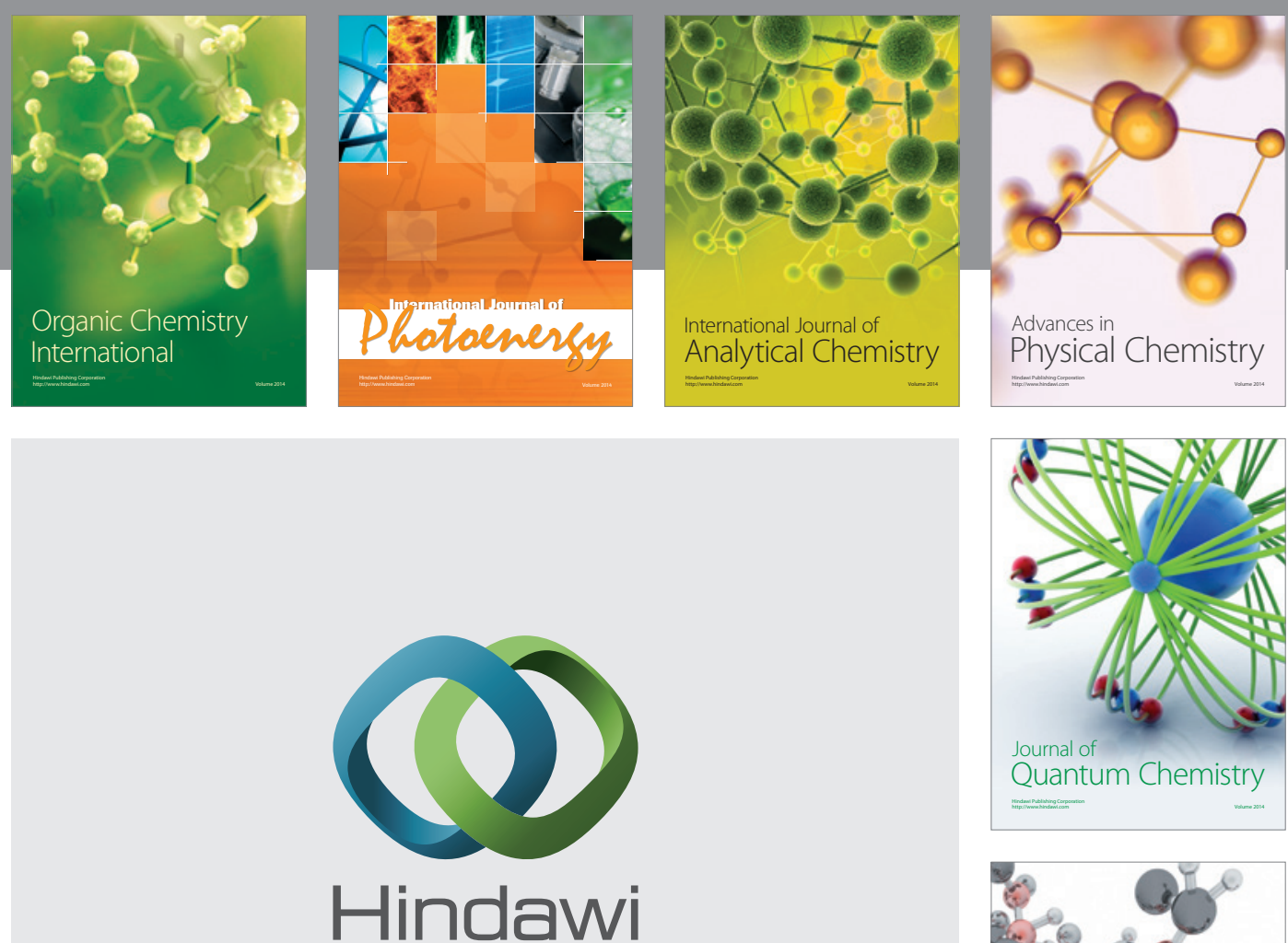

Submit your manuscripts at

http://www.hindawi.com

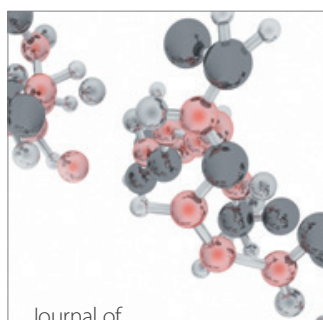

Analytical Methods

in Chemistry

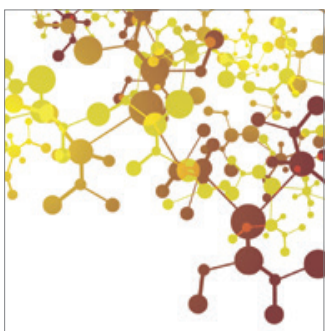

Journal of

Applied Chemistry

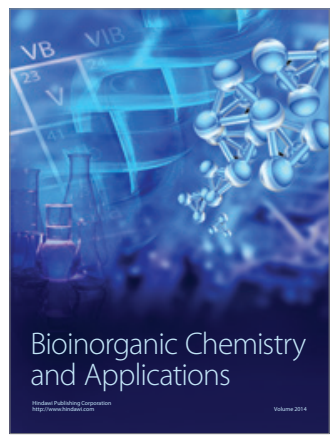

Inorganic Chemistry
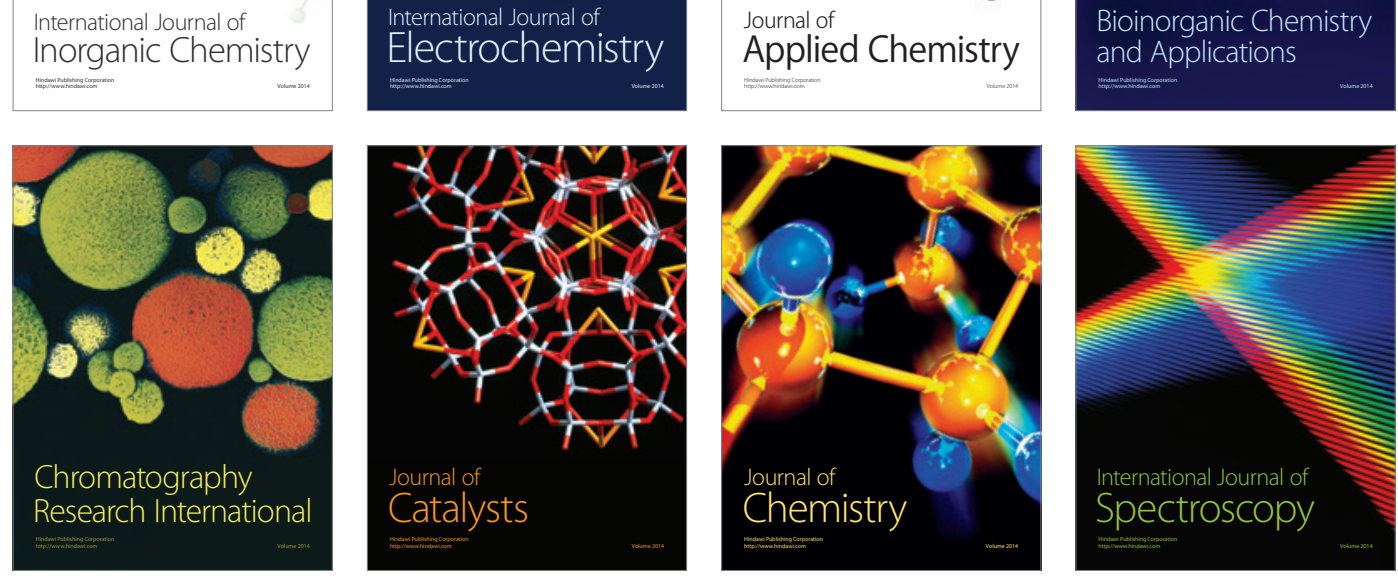